\title{
FINITE VOLUME METHODS FOR ELLIPTIC PDE'S: A NEW APPROACH
}

\author{
PAnAgiotis Chatzipantelidis ${ }^{1}$
}

\begin{abstract}
We consider a new formulation for finite volume element methods, which is satisfied by known finite volume methods and it can be used to introduce new ones. This framework results by approximating the test function in the formulation of finite element method. We analyze piecewise linear conforming or nonconforming approximations on nonuniform triangulations and prove optimal order $H^{1}$-norm and $L^{2}$-norm error estimates.
\end{abstract}

Mathematics Subject Classification. 65N30, 65N15.

Received: October 9, 2001. Revised: January 24, 2002.

\section{INTRODUCTION}

Finite volume method is a discretization technique widely used in the approximation of conservation laws, in computational fluid dynamics, in nonlinear problems in two or more dimensions, and in convection-diffusion problems see e.g. $[6,11,15,16,21,24]$. It can be viewed as a generalization of the finite difference method in triangular meshes. Apart from an approximation of the solution at discrete points, like in finite differences, we can seek an approximation in a finite element space. This version of the finite volume method is often called finite volume element method. It is possible to consider finite volume methods as an approximation of the finite element method through an appropriate quadrature rule, in 1-D, cf., e.g., Roos et al. [26, Chap. I, 2.3, p. 77], or in 2-D in quadrilateral meshes, $c f .$, e.g., Weiser and Wheeler [29].

We are interested in investigating a possible connection between the finite element and finite volume element methods using piecewise linear functions in triangular meshes, for second order elliptic boundary value problems. This will increase the understanding of these methods, will help us in the analysis of finite volumes and motivate us in developing new finite volume methods. Here we present a unified framework that describes known finite volume element methods and it provides a connection with the finite element method. In addition based on the proposed framework we construct new finite volume element methods having as control volumes the support of the basis functions of the corresponding finite element spaces.

We consider an elliptic boundary value problem of the form: Given $f \in L^{2}(\Omega)$, find a function $u: \Omega \rightarrow \mathbb{R}$ satisfying

$$
L u=f \quad \text { in } \Omega \text { and } \quad u=0 \quad \text { on } \partial \Omega,
$$

Keywords and phrases. Finite volume methods, error estimates

${ }^{1}$ Department of Applied Mathematics, University of Crete and Institute of Applied and Computational Mathematics, FO.R.T.H, 71110 Heraklion, Crete, Greece. e-mail: chatzipa@math.tamu.edu 
with $\Omega$ a bounded, convex, polygonal domain in $\mathbb{R}^{2}, L v \equiv-\operatorname{div}(A \nabla v), A=\left(a_{i j}\right)_{i, j=1}^{2}$ a given symmetric matrix function with real-value entries $a_{i j} \in W^{1, \infty}(\Omega), 1 \leq i, j \leq 2$. We assume that the matrix $A$ is uniformly positive definite, i.e., there exists a positive constant $\alpha_{0}$ such that

$$
\xi^{T} A(x) \xi \geq \alpha_{0} \xi^{T} \xi, \quad \forall \xi \in \mathbb{R}^{2}, \forall x \in \bar{\Omega} .
$$

Problem (1.1) can be equivalently written in the form

$$
a(u, v)=(f, v), \quad \forall v \in H_{0}^{1}(\Omega),
$$

where $a(v, w)=\sum_{K \in T_{h}} \int_{K} A \nabla v \cdot \nabla w \mathrm{~d} x,(\cdot, \cdot)$ the usual inner product in $L^{2}(\Omega), T_{h}$ a given triangulation of $\Omega$ and $h$ the maximum diameter of the triangles of $T_{h}$. Given a finite dimensional space $V_{h}, V_{h} \subset H_{0}^{1}(\Omega)$ (conforming elements), or $V_{h} \not \subset H_{0}^{1}(\Omega)$ (nonconforming elements), the finite element approximate solution $u_{h} \in V_{h}$ satisfies (1.3) with test functions in $V_{h}$, i.e.

$$
a\left(u_{h}, \chi\right)=(f, \chi), \quad \forall \chi \in V_{h} .
$$

Here we will restrict our attention to piecewise linear functions in $T_{h}$, thus $V_{h}$ will be either the standard finite element space of piecewise linear functions or the Crouzeix-Raviart finite element space, $c f$. [14].

In contrast to the finite element method which is based on a global variational form, finite volume methods rely on the local property of (1.1),

$$
-\int_{\partial \mathcal{O}}(A \nabla u) \cdot n \mathrm{~d} s=\int_{\mathcal{O}} f \mathrm{~d} x, \quad \forall \mathcal{O} \subset \Omega .
$$

In the finite volume method we seek an approximation $u_{h} \in V_{h}$ of $u$ which satisfies (1.5) on a finite collection $T_{h}{ }^{\prime}$ of polygonal domains $b \subset \Omega$, called control volumes;

$$
-\int_{\partial b}\left(A \nabla u_{h}\right) \cdot n \mathrm{~d} s=\int_{b} f \mathrm{~d} x, \quad \forall b \in T_{h}{ }^{\prime} .
$$

In order to construct an approximate solution of (1.1) the number of control volumes must be equal to the dimension of $V_{h}$. For these methods $H^{1}$ - and $L^{2}$-norm error estimates are known, cf., e.g. [2,5,19] and [12]. In addition in [19] there is a particularly interesting counterexample of a finite volume method which does not converge with optimal rate in the $L^{2}$-norm. However, in [12] and [15], optimal order $L^{2}$-norm convergence are proved if the control volumes are constructed using the barycenter of the triangles.

Finite volume schemes using nonconforming piecewise linear approximations were considered by the author in [7] and for Stokes problem in [11] and [20].

\section{New formulation}

It is known that in the case of Poisson's equation the corresponding bilinear forms of the finite element and finite volume method when using either conforming or nonconforming piecewise linear approximations, are identical, cf., e.g. [2, Lem. 3] and [7, Lem. 3.1 and 3.2]. In Section 3 we will show that these "classic" finite volume methods can be written in the form

$$
a\left(u_{h}, \chi\right)=\left(f, Q_{1} \chi\right), \quad \forall \chi \in V_{h},
$$

where $Q_{1} \chi$ is defined as an appropriate linear combination of point values of $\chi, c f .(3.2)$ and (3.6). 
However, in the general case of the operator $A$ with variable coefficients, (1.7) is no longer valid and matters are more complicated. Here we propose the following formulation

$$
a_{h}\left(u_{h}, \chi\right) \equiv \sum_{K \in T_{h}}\left\{\left(A \nabla u_{h} \cdot n, Q_{2}^{K} \chi\right)_{\partial K}+\left(L u_{h}, Q_{1} \chi\right)_{K}\right\}=\left(f, Q_{1} \chi\right)
$$

with $Q_{1}$ as in (1.7) and $Q_{2}^{K}$ similarly defined to $Q_{1}$. Then in Section 3 we show that the finite volume methods of [2,5], (conforming) and of [7] (nonconforming) can be written in this form, if $Q_{2}^{K} \equiv Q_{1}$. The formulation (1.8) covers also the finite element method since if $Q_{1}=Q_{2}^{K} \equiv 1$ we have

$$
\sum_{K \in T_{h}}\left\{\left(A \nabla u_{h} \cdot n, \chi\right)_{\partial K}+\left(L u_{h}, \chi\right)_{K}\right\}=a\left(u_{h}, \chi\right), \quad \forall \chi \in V_{h},
$$

with $(\cdot, \cdot)_{\partial K}$ and $(\cdot, \cdot)_{K}$ the usual inner products in $L^{2}(\partial K)$ and $L^{2}(K)$, respectively. In addition (1.8) is a generalization of (1.7). Indeed, in the case that $A$ is a $2 \times 2$ constant matrix, in view the required assumption $\int Q_{2}^{K} \chi=\int \chi$ over the sides of the triangles, $c f$. Section 6 , and that $L \chi=0$, for $\chi \in V_{h}$, we get

$$
a_{h}(\chi, \psi)=\sum_{K}(A \nabla \chi \cdot n, \psi)_{\partial K}=a(\chi, \psi), \quad \forall \chi, \psi \in V_{h} .
$$

The fact that the finite volume methods can be written in the general form of (1.7) and (1.8) clarifies the connection with the finite element method. Thus using this formulation we can significantly simplify known proofs and prove new results in $L^{2}$, without any assumption on the mesh other than regularity. Similar approaches where also developed in [12,15] and [23]. Note that according to our knowledge there is not another unified formulation that covers the analysis of conforming and nonconforming finite volume element methods.

\section{New finite volume methods}

The discrete method (1.8) leads to the introduction of new finite volume schemes, with the characteristic property of having as control volumes the support domains of the basis functions of the finite element space, (in this case the control volumes overlap), $c f$. Section 4 . These methods are clearly simpler to implement since the dual mesh is constructed using elements of the primal mesh. They admit a local form which in the case of constant coefficients with conforming piecewise linear approximations reads

$$
\int_{\partial b} \nabla u_{h} \cdot n \mathrm{~d} s=\frac{2}{3} \int_{b} f \mathrm{~d} x
$$

i.e., the discrete local conservation over the control volumes (1.6) is no longer satisfied, (a rather expected fact since the control volumes overlap). We show error estimates of optimal order in $H^{1}$ - and $L^{2}$-norm. An implicit conclusion of our results is that the finite volume scheme on these control volumes

$$
\int_{\partial b} \nabla u_{h} \cdot n \mathrm{~d} s=\int_{b} f \mathrm{~d} x,
$$

diverges. A rather surprising fact is the factor $\frac{2}{3}$ in (1.9) leads to a convergent method with optimal order. Similar results hold for the nonconforming case.

\section{New convergence results}

Even though $H^{1}$-norm error estimates are optimal in order and in regularity, the $L^{2}$-norm error estimates require additional regularity assumptions for the exact solution than the finite element method, i.e., $u \in$ $W^{3, p}(\Omega), c f$. [12] and [15]. In Section 5 we show that the solution $u_{h}$ of (1.8) satisfies optimal order $H^{1}-$ and $L^{2}$-norm error estimates. The $L^{2}$-norm estimate holds if $f \in W^{1, p}, 1<p \leq 2, A \in W^{2, \infty}$ and $\int Q_{1}$ defines a 
quadrature rule for area integrals exact for piecewise linear functions. Thus, we derive $L^{2}$-norm error estimates with $u \in H^{2}$ and additional assumptions posed only on the data $f$. Similar error estimates were derived by Hackbusch, in [18], in the case of a diagonal matrix function $A$. Note that the error analysis presented here works for both conforming and nonconforming finite element spaces.

\section{Extensions}

- New methods. In principle the proposed formulation (1.8) is not restricted to the finite dimensional spaces $V_{h}$ of piecewise linear functions. Thus using different finite dimensional spaces $V_{h}$ and operators $Q_{1}$ and $Q_{2}^{K}$, than the ones proposed here, we can construct new methods, possibly better than the ones considered here.

- High order methods-Overlapping. The analysis of finite volume methods with nonoverlapping control volumes, using piecewise polynomials of degree greater than one is involved and restricted, to our knowledge, to quadratic polynomials, $c f$. [22]. One reason is that the construction of the dual mesh becomes more complex as the degree of the polynomial approximation increases. However, the use of the support of the basis functions as control volumes seems to overcome this problem. The general approach, suggested here, has been used by Plexousakis and Zouraris in [25] for the construction and analysis of conservative high order finite volume methods.

- New results. Based on the formulation (1.8) we can derive error estimates in various problems related to (1.1). Thus, for the Stokes problem, the a posteriori error estimators for the $H^{1}-$ and $L^{2}-$ norm are similar to the ones used in finite element method, e.g. for nonconforming piecewise linear approximations, $c f$. [10]. Also, known error estimates for the parabolic problem can be improved, regarding the required regularity of the exact solution, $c f$. [8]. In addition note that in the case of a nonconvex polygonal domain $\Omega$ the exact solution $u$ of (1.1) will not be $W^{3, p}(\Omega), p>1, c f .$, e.g. [17, Chap. 5]. In [9], we derive $H^{1}$ - and $L^{2}$-norm error estimates for (1.1), where $\Omega$ is a nonconvex polygonal domain, with minimum assumptions on the exact solution $u$.

An outline of the paper is the following. In Section 2 we introduce notation and in Section 3 we show that the classic finite volume methods can be written in the formulation (1.8). In Section 4 we describe new methods with overlapping control volumes whose construction is motivated by (1.8). Then in Sections 5 and 6 , under sufficient assumptions for $Q_{1}$ and $Q_{2}^{K}$, we analyze the discrete scheme (1.8) and prove optimal order error estimates.

\section{Preliminaries}

Consider a bounded, convex polygonal domain $\Omega$ and a triangulation $T_{h}$ of $\Omega$. Let $h_{K}$ be the diameter of the triangle $K \in T_{h}$ and $h=\max _{K \in T_{h}} h_{K}$. We assume that the family $\left\{T_{h}\right\}_{0<h<1}$ of triangulations is nondegenerate, i.e. there exists a positive constant $\sigma$, independent of $h$, such that, for every $K \in T_{h}, \rho_{K} \geq \sigma h_{K}$, with $\rho_{K}$ denoting the diameter of the largest circle contained in $K \in T_{h}$, see, e.g. [3, p. 106]. This assumption is equivalent to the minimal angle condition.

Given a domain $K \subset \mathbb{R}^{2}$, denote by $L^{2}(K)$ the space of square integrable real functions over $K,(\cdot, \cdot)_{K}$ the inner product in $L^{2}(K),|\cdot|_{s, K}$ and $\|\cdot\|_{s, K}$ the seminorm and norm, respectively, of the Sobolev space $H^{s}(K),|\cdot|_{W^{s, p}(K)}$ and $\|\cdot\|_{W^{s, p}(K)}$ the seminorm and norm, respectively, of the Sobolev space $W^{s, p}(K), s \in \mathbb{N}$, $p \geq 1$. Then, we introduce the norm $\|\cdot\|_{s, h},\|v\|_{s, h}=\left\{\sum_{K}\|v\|_{s, K}^{2}\right\}^{1 / 2}$, and the seminorm $|\cdot|_{s, h},|v|_{s, h}=$ $\left\{\sum_{K}|v|_{s, K}^{2}\right\}^{1 / 2}, s \in \mathbb{N}$. In addition, if $K=\Omega$ we suppress the index $K$, and if $s=0$ we suppress the index $s$. Also with $|\cdot|$ we denote either the area of a region in $\mathbb{R}^{2}$ or the length of a line segment, and with $\|\cdot\|_{\mathbb{R}^{2}}$ the Euclidean norm on $\mathbb{R}^{2}$ and also the subordinate matrix norm.

In the sequel we introduce notation. Given a triangle $K \in T_{h}$, let $Z_{h}(K)$ and $E_{h}(K)$ be the set of the vertices and sides, respectively, of $K, Z_{h}=\cup_{K \in T_{h}} Z_{h}(K)$ and $E_{h}=\cup_{K \in T_{h}} E_{h}(K)$. Next, let $Z_{h}^{\text {in }}$ and $E_{h}^{\text {in }}$ be the set of the interior vertices and sides, respectively, of $T_{h}$ and denote by $m_{e}$ the middle point of a side $e \in E_{h}$. 

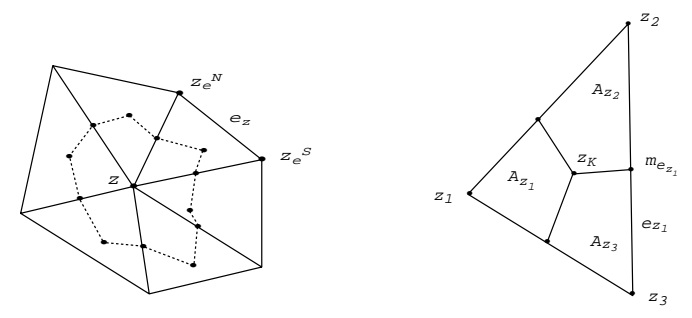

Figure 1. Left: A sample region $V_{z}$. With dotted lines the corresponding box $b_{z}$. Right: A triangle $\mathrm{K}$ partitioned into three subregions $A_{z}$.
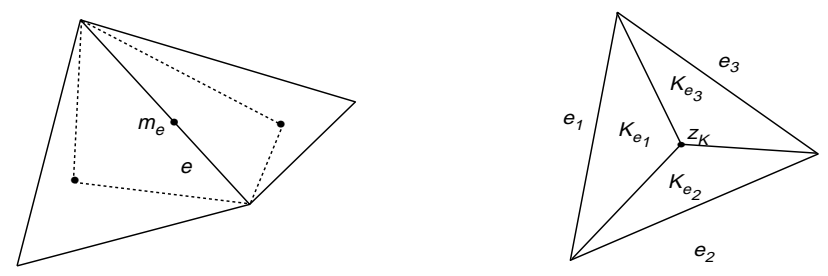

Figure 2. Left: A sample region $V_{e}$. With dotted lines the corresponding box $b_{e}$. Right: A triangle $\mathrm{K}$ partitioned into three subtriangles $K_{e}$.

Next we will consider the finite collections of control volumes $\mathcal{V}_{c}, \mathcal{B}_{c}, \mathcal{V}_{n}$ and $\mathcal{B}_{n}$. With each vertex $z \in Z_{h}^{\text {in }}$ we associate a region $V_{z} \subset \Omega$, consisting of those triangles $K \in T_{h}$ that have $z$ as a vertex and denote $\mathcal{V}_{c}=\left\{V_{z}: z \in Z_{h}^{\text {in }}\right\}$.

We construct $\mathcal{B}_{c}$ in the following way: For each triangle $K \in T_{h}$ consider an interior point $z_{K}$. Connect $z_{K}$ with line segments to the edge midpoints of $K$. This partitions $K$ into three subregions $A_{z}, z \in Z_{h}(K)$, see Figure 1. Let $\mathcal{A}(K)=\left\{A_{z}: z \in Z_{h}(K)\right\}$. With each vertex $z \in Z_{h}^{\text {in }}$ we associate a box $b_{z} \subset V_{z}$, which consists of the union of the subregions $A_{z}$ in $V_{z}$, which have $z$ as a corner, see Figure 1 , and let $\mathcal{B}_{c}=\left\{b_{z}: z \in Z_{h}^{\text {in }}\right\}$.

Also, with any $e \in E_{h}^{\text {in }}$ we associate a region $V_{e}$, consisting of the two triangles of $T_{h}$ with common side $e$, and let $\mathcal{V}_{n}=\left\{V_{e}: e \in E_{h}^{\text {in }}\right\}$, see Figure 2 .

Finally consider an interior point $z_{K}$ of $K \in T_{h}$ and connect it with line segments to the vertices of $K$. Thus we partition $K$ to three subtriangles $K_{e}, e \in E_{h}(K)$. Let $\tilde{T}_{h}(K)$ be the set $\left\{K_{e}: e \in E_{h}(K)\right\}$. Now, with each side $e \in E_{h}^{\text {in }}$ we associate a box $b_{e} \subset V_{e}$ consisting of the two subtriangles $K_{e}$, in $V_{e}$, which have $e$ as a common side and let $\mathcal{B}_{n}=\left\{b_{e}: e \in E_{h}^{\text {in }}\right\}$.

Consider now, the finite dimensional space $X_{h}^{0}=\left\{\chi \in C(\Omega):\left.\chi\right|_{K}\right.$ is linear for all $K \in T_{h}$ and $\left.\left.\chi\right|_{\partial \Omega}=0\right\}$ and the finite dimensional space $S_{h}^{0}$ which consists of the piecewise linear functions in $K \in T_{h}$ that are continuous at the middle points of a side $e \in E_{h}, m_{e}$, and $\chi\left(m_{e}\right)=0$ if $e \in E_{h} \backslash E_{h}^{\text {in }}$. Note that $S_{h}^{0}$ is not a subspace of $H_{0}^{1}$. In general we will denote $X_{h}^{0}$ and $S_{h}^{0}$ with $V_{h}$.

We have that $X_{h}^{0}$ and $S_{h}^{0}$ satisfy the following approximation property

$$
\inf _{\chi \in V_{h}}\|v-\chi\|_{j, h} \leq C\left(\sum_{K \in T_{h}} h_{K}^{2(2-j)}\|v\|_{2, K}^{2}\right)^{1 / 2}, \quad \forall v \in H^{2} \cap H_{0}^{1}, j=0,1,
$$

cf., e.g. [13, Th. 15.3].

\section{Applications I: Classic finite volume methods}

In this section we consider two "classic" finite volume schemes for (1.1), using as an approximation space conforming or nonconforming piecewise linear functions on a given triangulation $T_{h}$. Then for appropriate 
choices of operator $Q_{1}$ given by (3.2) and (3.6), respectively, we can rewrite them in the form (1.8). Therefore, the error estimates proved for (1.8) in Section 5, $c f$. Theorems 5.1 and 5.2, will also hold for these methods.

Method I: We consider the finite volume approximations in $X_{h}^{0}$ of (1.1), with control volumes in $\mathcal{B}_{c}$, i.e. Find $u_{h} \in X_{h}^{0}$ such that

$$
-\int_{\partial b_{z}}\left(A \nabla u_{h}\right) \cdot n \mathrm{~d} s=\int_{b_{z}} f \mathrm{~d} x, \quad \forall z \in Z_{h}^{\text {in }},
$$

which can be equivalently rewritten

$$
\bar{a}_{C N}\left(u_{h}, \chi\right)=\sum_{z \in Z_{h}^{\text {in }}} \chi(z) \int_{b_{z}} f \mathrm{~d} x, \quad \forall \chi \in X_{h}^{0},
$$

with $\bar{a}_{C N}(\cdot, \cdot): H_{h}^{2} \times H_{h}^{2} \rightarrow \mathbb{R}$ defined by $\bar{a}_{C N}(v, w)=-\sum_{z \in Z_{h}^{\text {in }}} w(z) \int_{\partial b_{z}}(A \nabla v) \cdot n \mathrm{~d} s$ and $H_{h}^{2}=H^{2}+X_{h}^{0}$.

Next, we will show that this discrete scheme can be written in the form (1.8). Let us consider the linear operator $\left.Q_{1}\right|_{K}: \mathbb{P}_{1}(K) \rightarrow L^{2}(K)$ defined by,

$$
\left.Q_{1} \chi\right|_{K}=\sum_{z \in Z_{h}(K)} \chi(z) q_{A_{z}}
$$

with $q_{S}$ the characteristic function of $S$ and $A_{z} \in \mathcal{A}(K)$ the regions in which we partition $K \in T_{h}$, see Figure 1 . Then, for every $f \in L^{2}$ and $\chi \in X_{h}^{0}$, it is easily seen that

$$
\left(f, Q_{1} \chi\right)=\sum_{K} \sum_{z \in Z_{h}(K)} \chi(z) \int_{A_{z}} f \mathrm{~d} x=\sum_{z \in Z_{h}^{\text {in }}} \chi(z) \int_{b_{z}} f \mathrm{~d} x .
$$

In the case of Poisson's equation it is known, $c f .[2$, Lem. 3$]$, that

$$
a(\chi, \psi)=\bar{a}_{C N}(\chi, \psi), \quad \forall \chi, \psi \in X_{h}^{0} .
$$

Thus, in view of (3.3) the finite volume method (3.1) can be written equivalently in the form (1.7). In the general case now, by integration by parts we obtain

$$
\int_{A_{z}} L \chi \mathrm{d} x+\int_{\partial A_{z} \cap \partial K} A \nabla \chi \cdot n \mathrm{~d} s=-\int_{\partial A_{z} \cap \partial b_{z}} A \nabla \chi \cdot n \mathrm{~d} s, \quad \forall \chi \in X_{h}^{0},
$$

with $z \in Z_{h}$ and $K \in T_{h}$. Multiplying then with $\psi(z), \psi \in X_{h}^{0}$, and summing subsequently over the triangles that have $z$ as a common vertex and the vertices $z \in Z_{h}^{\text {in }}$, we get $a_{h}(\chi, \psi)=\bar{a}_{C N}(\chi, \psi)$. Hence, the solution $u_{h}$ of (3.1) satisfies (1.8).

Method II: In [7] we consider finite volume approximations for (1.1) in $S_{h}^{0}$, with control volumes in $\mathcal{B}_{n}$, i.e., find $u_{h} \in S_{h}^{0}$ such that

$$
-\int_{\partial b_{e}}\left(A \nabla u_{h}\right) \cdot n \mathrm{~d} s=\int_{b_{e}} f \mathrm{~d} x, \quad \forall e \in E_{h}^{\mathrm{in}},
$$

which can be equivalently rewritten

$$
\bar{a}_{N N}\left(u_{h}, \chi\right)=\sum_{e \in E_{h}^{\text {in }}} \chi\left(m_{e}\right) \int_{b_{e}} f \mathrm{~d} x, \quad \forall \chi \in S_{h}^{0},
$$


with $\bar{a}_{N N}(\cdot, \cdot): \tilde{H}_{h}^{2} \times \tilde{H}_{h}^{2} \rightarrow \mathbb{R}$ defined by $\bar{a}_{N N}(v, w)=-\sum_{e \in E_{h}^{\text {in }}} w\left(m_{e}\right) \int_{\partial b_{e}}(A \nabla v) \cdot n \mathrm{~d} s$ and $\tilde{H}_{h}^{2}=H^{2}+S_{h}^{0}$.

Similarly, as in Method I, we can show that this discrete scheme can be written in the form (1.8), where the linear operator $\left.Q_{1}\right|_{K}: \mathbb{P}_{1}(K) \rightarrow L^{2}(K)$ is defined by

$$
\left.Q_{1} \chi\right|_{K}=\sum_{e \in E_{h}(K)} \chi\left(m_{e}\right) q_{K_{e}}
$$

with $K_{e} \in \tilde{T}_{h}(K)$ are the regions we partition $K \in T_{h}$ see Figure 2 and $q_{S}$ the characteristic function of a set $S \subset \mathbb{R}^{2}$.

Remark 3.1. If in the construction of the control volumes $\mathcal{B}_{c}$ and $\mathcal{B}_{c}$ we choose $z_{K}$ to be the barycenter of $K \in T_{h}$, we have

$$
\int_{K} Q_{1} \chi \mathrm{d} x=\int_{K} \chi \mathrm{d} x, \quad \forall \chi \in \mathbb{P}_{1}(K)
$$

with $Q_{1}$ defined by either (3.2) or (3.6). Therefore, $\int Q_{1}$ defines a quadrature rule for area integrals which is exact for linear functions.

\section{ApPlications II: OVERLAPPing CONTROL VOLUMES}

In this section we consider two new finite volume schemes for (1.1) with control volumes in $\mathcal{V}_{c}$ and $\mathcal{V}_{n}$, using as an approximation space conforming and nonconforming piecewise linear functions, respectively, on a given triangulation $T_{h}$. For appropriate choices of operators $Q_{1}$ and $Q_{2}^{K}$ we can rewrite them in the form (1.8). In contrast to Methods I and II, here the solution $u$ of (1.1) and its approximation $u_{h}$ do not satisfy the same local conservation property, cf., e.g., (4.4) and (4.5). However, the construction of the dual mesh is simpler. This approach helps in developing new high order finite volume methods, $c f$. [25].

In Method III we seek $u_{h} \in X_{h}^{0}$ such that

$$
-\int_{\partial V_{z}}\left(A \nabla u_{h}\right) \cdot n \mathrm{~d} s+\frac{1}{2} \sum_{e \in E_{h}^{\star}\left(V_{z}\right)} \int_{e}\left[A \nabla u_{h}\right] \cdot n_{e} \mathrm{~d} s=\int_{V_{z}} f \mathrm{~d} x, \quad \forall z \in Z_{h}^{\mathrm{in}}
$$

and in Method IV we seek $u_{h} \in S_{h}^{0}$ such that

$$
-\int_{\partial V_{e}}\left(A \nabla u_{h}\right) \cdot n \mathrm{~d} s+2 \int_{e}\left[A \nabla u_{h}\right] \cdot n_{e} \mathrm{~d} s=\int_{V_{e}} f \mathrm{~d} x, \quad \forall e \in E_{h}^{\mathrm{in}},
$$

where

$$
[A \nabla \chi] \cdot n_{e}=\left(A \nabla \chi_{K^{+e}}\right) \cdot n_{e}-\left(A \nabla \chi_{K^{-e}}\right) \cdot n_{e}
$$

with $K^{+e}, K^{-e}$ denoting two triangles having $e \in E_{h}^{\text {in }}$ as a common edge and $n_{e}$ the normal vector of $e \in$ $E_{h}\left(K^{+e}\right), c f$. Figure 3 , and $E_{h}^{\star}\left(V_{z}\right)=\left\{e \in E_{h}: z \in e\right\}, c f$. Figure 3 .

Method III: We consider finite volume approximations in $X_{h}^{0}$ with control volumes in $\mathcal{V}_{c}$. As a first step we will construct the method for Poisson's equation and then for the general equation (1.1).

The balance equation for Poisson's equation over the control volume $V_{z} \in \mathcal{V}_{c}$ is

$$
-\int_{\partial V_{z}} \nabla u \cdot n \mathrm{~d} s=\int_{V_{z}} f \mathrm{~d} x .
$$



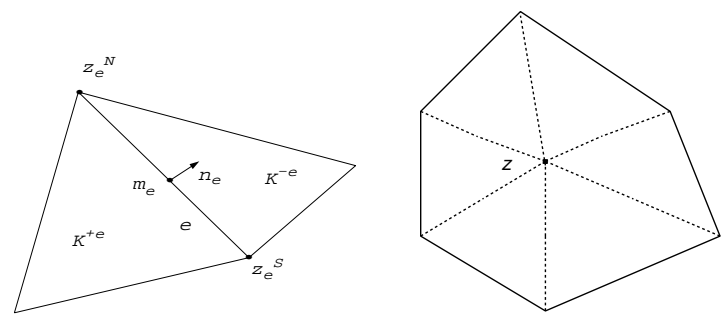

Figure 3. Left: The triangles $K^{+e}$ and $K^{-e}$ with common side e. Right: A box $V_{z}$; with dotted lines the sides of the set $E_{h}^{\star}\left(V_{z}\right)$.

The appropriate finite volume method in this case is: Find $u_{h} \in X_{h}^{0}$ such that

$$
-\int_{\partial V_{z}} \nabla u_{h} \cdot n \mathrm{~d} s=\frac{2}{3} \int_{V_{z}} f \mathrm{~d} x, \quad \forall z \in Z_{h}^{\text {in }},
$$

which can be equivalently rewritten

$$
\bar{a}_{C O}\left(u_{h}, \chi\right)=\frac{2}{3} \sum_{z \in Z_{h}^{\text {in }}} \chi(z) \int_{V_{z}} f \mathrm{~d} x
$$

where $\bar{a}_{C O}(\cdot, \cdot): H_{h}^{2} \times H_{h}^{2} \rightarrow \mathbb{R}$ is defined by $\bar{a}_{C O}(v, w)=-\sum_{z \in Z_{h}^{\text {in }}} w(z) \int_{\partial V_{z}} \nabla v \cdot n \mathrm{~d} s$.

For this method the corresponding relation to (3.4) does not hold; however we can prove the following lemma.

Lemma 4.1. Let $\chi, \psi \in X_{h}^{0}$. Then

$$
\bar{a}_{C O}(\chi, \psi)=2 a(\chi, \psi) .
$$

Proof. Let $\left\{\omega_{z}\right\}_{z \in Z_{h}}$ be the usual basis of $X_{h}^{0}$, where $\omega_{z}$ is one on vertex $z$ and zero in every other. Then, in order to get the desired relation it is sufficient to show it for $\psi=\omega_{z}$. For $z \in K$ and $\chi \in X_{h}^{0}$, we have

$$
\left(\nabla \chi, \nabla \omega_{z}\right)_{K}=\int_{\partial K} \omega_{z} \nabla \chi \cdot n \mathrm{~d} s=\sum_{e \in E_{h}(K)} \nabla \chi \cdot n \int_{e} \omega_{z} \mathrm{~d} s
$$

Since $\omega_{z}$ is a linear polynomial on every side of $K$, we get

$$
\int_{e} \omega_{z} \mathrm{~d} s=\frac{|e|}{2}\left(\omega_{z}\left(z_{e}^{S}\right)+\omega_{z}\left(z_{e}^{N}\right)\right), \quad \forall e \in E_{h}(K),
$$

where by $|e|$ we denote the length of $e$. Therefore,

$$
\int_{e_{z}} \omega_{z} \mathrm{~d} s=0 \text { and } \int_{\ell} \omega_{z} \mathrm{~d} s=\frac{|e|}{2}, \quad \text { for } \ell \in E_{h}(K) \backslash\left\{e_{z}\right\},
$$

with $e_{z}$ the side of $K$ opposite to $z$, see Figure 1. Combining now (4.6)-(4.8), we easily obtain

$$
\int_{K} \nabla \chi \cdot \nabla \omega_{z} \mathrm{~d} x=\frac{1}{2} \sum_{e \in E_{h}(K) \backslash\left\{e_{z}\right\}} \int_{e} \nabla \chi \cdot n \mathrm{~d} s=-\frac{1}{2} \int_{e_{z}} \nabla \chi \cdot n \mathrm{~d} s
$$


Next, let us consider the linear operator $\left.Q_{1}\right|_{K}: \mathbb{P}_{1}(K) \rightarrow L^{2}(K)$ defined by

$$
\left.Q_{1} \chi\right|_{K}=\frac{1}{3} \sum_{z \in Z_{h}(K)} \chi(z)
$$

with $K \in T_{h}$ and $\chi \in X_{h}^{0}$. We can easily see for every $f \in L^{2}$ and $\chi \in X_{h}^{0}$

$$
\left(f, Q_{1} \chi\right)=\frac{1}{3} \sum_{K} \sum_{z \in Z_{h}(K)} \chi(z) \int_{K} f \mathrm{~d} x=\frac{1}{3} \sum_{z \in Z_{h}^{\text {in }}} \chi(z) \int_{V_{z}} f \mathrm{~d} x .
$$

Thus, (4.5) can be written equivalently in the form (1.7).

In the general case of equation (1.1) the obvious generalization of (4.5) does not seem to be the correct one. In order to derive the "correct" method with control volumes in $\mathcal{V}_{c}$ we will consider appropriate operators $Q_{1}$ and $Q_{2}^{K}$ in the discrete method (1.8). Let $Q_{1}$ be defined the same way as above and $Q_{2}^{K}$ be a linear operator $\left.Q_{2}^{K}\right|_{e}: \mathbb{P}_{1}(K) \rightarrow L^{2}(e)$, be defined by

$$
\left.Q_{2}^{K} \chi\right|_{e}=\frac{1}{2}\left(\chi\left(z_{e}^{N}\right)+\chi\left(z_{e}^{S}\right)\right)
$$

with $e \in E_{h}(K)$, and $z_{e}^{N}$ and $z_{e}^{S}$ the two vertices of the $e$, see Figure 3 . Then, the bilinear form $a_{h}$ can be written in the form

$$
a_{h}(\chi, \psi)=\frac{1}{2} \sum_{K} \sum_{e \in E_{h}(K)}\left(A \nabla \chi \cdot n, \psi\left(z_{e}^{N}\right)+\psi\left(z_{e}^{S}\right)\right)_{e}+\frac{1}{3} \sum_{z \in Z_{h}^{\text {in }}} \psi(z) \sum_{K \in T_{h}\left(V_{z}\right)} \int_{K} L \chi \mathrm{d} x
$$

with $T_{h}\left(V_{z}\right)$ denoting the triangles forming $V_{z}$.

Let us consider now a triangle $K \in T_{h}$ and a vertex $z \in Z_{h}(K) \cap Z_{h}^{\text {in }}$; by integration by parts we obtain

$$
\int_{K} L \chi \mathrm{d} x+\frac{3}{2} \int_{\partial K \backslash \partial V_{z}} A \nabla \chi \cdot n \mathrm{~d} s=-\int_{\partial K \cap \partial V_{z}} A \nabla \chi \cdot n \mathrm{~d} s+\frac{1}{2} \int_{\partial K \backslash \partial V_{z}} A \nabla \chi \cdot n \mathrm{~d} s .
$$

Multiplying then with $\psi(z), \psi \in X_{h}^{0}$, and summing subsequently over the triangles that have $z$ as a common vertex and the vertices $z \in Z_{h}^{\text {in }}$, we get

$$
3 a_{h}(\chi, \psi)=\bar{a}_{C O}(\chi, \psi)+\frac{1}{2} \sum_{z \in Z_{h}^{\text {in }}} \psi(z) \sum_{e \in E_{h}^{\star}\left(V_{z}\right)} \int_{e}[A \nabla \chi] \cdot n_{e} \mathrm{~d} s
$$

with $[A \nabla \chi] \cdot n_{e}$ and $n_{e}$ as in $(4.3), E_{h}^{\star}\left(V_{z}\right)=\left\{e \in E_{h}: z \in e\right\}, c f$. Figure 3 and $\bar{a}_{C O}(\cdot, \cdot): H_{h}^{2} \times H_{h}^{2} \rightarrow \mathbb{R}$ defined by $\bar{a}_{C O}(v, w)=-\sum_{z \in Z_{h}^{\text {in }}} w(z) \int_{\partial V_{z}}(A \nabla v) \cdot n \mathrm{~d} s$. Thus the relation above and (4.10) suggest that the method (1.8) can be written equivalently in the form (4.1).

Method IV: This time we consider finite volume approximations in $S_{h}^{0}$ with control volumes in $\mathcal{V}_{n}$. As we did in Method III, we develop this method first for Poisson's equation. The balance equation over the control volume $V_{e} \in \mathcal{V}_{n}$ is

$$
-\int_{\partial V_{e}} \nabla u \cdot n \mathrm{~d} s=\int_{V_{e}} f \mathrm{~d} x .
$$

The appropriate finite volume method in this case is: Find $u_{h} \in S_{h}^{0}$ such that

$$
-\int_{\partial V_{e}} \nabla u_{h} \cdot n \mathrm{~d} s=\frac{1}{3} \int_{V_{e}} f \mathrm{~d} x, \quad \forall e \in E_{h}^{\mathrm{in}},
$$


which can be equivalently rewritten

$$
\bar{a}_{N O}\left(u_{h}, \chi\right)=\frac{1}{3} \sum_{e \in E_{h}^{\text {in }}} \chi\left(m_{e}\right) \int_{V_{e}} f \mathrm{~d} x, \quad \forall \chi \in S_{h}^{0},
$$

with $\bar{a}_{N O}(\cdot, \cdot): \tilde{H}_{h}^{2} \times \tilde{H}_{h}^{2} \rightarrow \mathbb{R}$ defined by $\bar{a}_{N O}(v, w)=-\sum_{e \in E_{h}^{\text {in }}} w\left(m_{e}\right) \int_{\partial V_{e}} \nabla v \cdot n \mathrm{~d} s$.

Using Green's formula and the fact that $\left.\chi\right|_{K} \in \mathbb{P}_{1}$, for every $\chi \in S_{h}^{0}$, we can prove

$$
\bar{a}_{N N}(\chi, \psi)=\bar{a}_{N O}(\chi, \psi), \quad \forall \chi, \psi \in S_{h}^{0} .
$$

Hence, in view of the fact that $\bar{a}_{N N}(\chi, \psi)=\bar{a}_{N O}(\chi, \psi)$, for every $\chi, \psi \in S_{h}^{0}, c f$. [7, Lem. 3.1 and 3.2], we have $\bar{a}_{N O}(\chi, \psi)=a(\chi, \psi)$.

Next, let us consider the linear operator $\left.Q_{1}\right|_{K}: \mathbb{P}_{1}(K) \rightarrow L^{2}(K)$ defined by

$$
\left.Q_{1} \chi\right|_{K}=\frac{1}{3} \sum_{e \in E_{h}(K)} \chi\left(m_{e}\right)
$$

with $K \in T_{h}$. We can easily see for every $f \in L^{2}$ and $\chi \in S_{h}^{0}$

$$
\left(f, Q_{1} \chi\right)=\frac{1}{3} \sum_{K} \int_{K} f \sum_{e \in E_{h}(K)} \chi\left(m_{e}\right) \mathrm{d} x=\frac{1}{3} \sum_{e \in E_{h}^{\text {in }}} \chi\left(m_{e}\right) \int_{V_{e}} f \mathrm{~d} x .
$$

Thus, (4.12) can be written equivalently in the form (1.7).

In the general case of equation (1.1) the obvious generalization of (4.12) does not seem to be the correct one. In order to derive the "correct" method with control volumes in $\mathcal{V}_{n}$ we will consider appropriate operators $Q_{1}$ and $Q_{2}^{K}$ in the discrete method (1.8). Let $Q_{1}$ be defined as above and the linear operator $\left.Q_{2}^{K}\right|_{e}: \mathbb{P}_{1}(K) \rightarrow L^{2}(e)$, be defined by

$$
\left.Q_{2}^{K} \chi\right|_{e}=\chi\left(m_{e}\right)
$$

with $e \in E_{h}(K)$. Then the bilinear form $a_{h}$ can be written in the form

$$
a_{h}(\chi, \psi)=\sum_{K} \sum_{e \in E_{h}(K)}\left(A \nabla \chi \cdot n, \psi\left(m_{e}\right)\right)_{e}+\frac{1}{3} \sum_{e \in E_{h}^{\text {in }}} \psi(z) \sum_{K \in T_{h}\left(V_{e}\right)} \int_{K} L \chi \mathrm{d} x,
$$

with $T_{h}\left(V_{e}\right)$ denoting the triangles with common edge $e$. Let us consider now a triangle $K \in T_{h}$ and an edge $e \in E_{h}(K) \cap E_{h}^{\text {in }}$; by integration by parts we obtain

$$
\int_{K} L \chi \mathrm{d} x+3 \int_{e} A \nabla \chi \cdot n \mathrm{~d} s=-\int_{\partial K \cap \partial V_{e}} A \nabla \chi \cdot n \mathrm{~d} s+2 \int_{e} A \nabla \chi \cdot n \mathrm{~d} s .
$$

Multiplying then with $\psi\left(m_{e}\right), \psi \in S_{h}^{0}$, and summing subsequently over the triangles that have $e$ as a common edge and the vertices $e \in E_{h}^{\text {in }}$, we get

$$
3 a_{h}(\chi, \psi)=\bar{a}_{N O}(\chi, \psi)+2 \sum_{E \in E_{h}^{\text {in }}} \psi\left(m_{e}\right) \int_{e}[A \nabla \chi] \cdot n_{e} \mathrm{~d} s, \quad \forall \chi, \psi \in S_{h}^{0},
$$

with $[A \nabla \chi] \cdot n_{e}$ and $n_{e}$ as in $(4.3)$ and $\bar{a}_{N O}(\cdot, \cdot): \tilde{H}_{h}^{2} \times \tilde{H}_{h}^{2} \rightarrow \mathbb{R}$ defined by $\bar{a}_{N O}(v, w)=-\sum_{e \in E_{h}^{\text {in }}} w\left(m_{e}\right) \int_{\partial V_{e}}$ $(A \nabla v) \cdot n \mathrm{~d} s$. Thus, the relation above and (4.14) suggest that the method (1.8) can be written equivalently in the form (4.2). 
Remark 4.1. In contrast to Methods I and II the discrete schemes considered here do not satisfy the same local balance equation as the continuous problem. An issue to examine is the coefficients $2 / 3$ and $1 / 3$ on the right-hand side of (4.5) and (4.12), respectively. Motivated by the discussion in [4], we can see that the scheme (4.5) satisfies a discrete analog of the conservation property (1.5) in a dilation of the control volume $V_{z}$ with ratio $\lambda=2 / 3$, i.e., let us assume that $f$ is constant, and let $V_{z, 2 / 3}=\left\{z+(2 / 3)(x-z): x \in V_{z}\right\}$. Then if $u_{h}$ satisfies $(4.5)$,

$$
-\int_{\partial V_{z, 2 / 3}} \nabla u_{h} \cdot n \mathrm{~d} s=-\frac{2}{3} \int_{\partial V_{z}} \nabla u_{h} \cdot n \mathrm{~d} s=\left(\frac{2}{3}\right)^{2} \int_{V_{z}} f \mathrm{~d} x=\int_{V_{z, 2 / 3}} f \mathrm{~d} x
$$

$c f$. Schmidt [27], for a finite volume method with overlapping control volumes that preserves local conservation. Similarly, we can see that the scheme (4.12) satisfies a discrete version of the conservation property (1.5) in a dilation of the control volume $V_{e}$ with ratio $\lambda=1 / 3$.

Remark 4.2. It is obvious that for $Q_{1}$ defined by either (4.9) or (4.13), $\int Q_{1}$ defines a quadrature rule for area integrals exact for linear functions, i.e.

$$
\int_{K} Q_{1} \chi \mathrm{d} x=\int_{K} \chi \mathrm{d} x, \quad \forall \chi \in \mathbb{P}_{1}(K) .
$$

\section{A General DisCREte PROBLEM}

In this section we will analyze the discrete method (1.8), and derive error estimates in $H^{1}-$ and $L^{2}-$ norm. Then, in view of the previous two sections, these error estimates will also hold for Methods I-IV.

We consider the problem: Find $u_{h} \in V_{h}$ such that

$$
a_{h}\left(u_{h}, \chi\right)=\left(f, Q_{1} \chi\right), \quad \forall \chi \in V_{h},
$$

with $a_{h}(\cdot, \cdot): V_{h} \times V_{h} \rightarrow \mathbb{R}$ defined by $a_{h}(\chi, \psi)=\sum_{K}\left\{\left(A \nabla \chi \cdot n, Q_{2}^{K} \psi\right)_{\partial K}+\left(L \chi, Q_{1} \psi\right)_{K}\right\}$.

Essential part in our analysis have the errors $\varepsilon_{h}$ and $\varepsilon_{a}$

$$
\begin{aligned}
& \varepsilon_{h}(f, \psi)=(f, \psi)-\left(f, Q_{1} \psi\right), \quad \forall f \in L^{2}, \psi \in V_{h}, \\
& \varepsilon_{a}(\chi, \psi)=a(\chi, \psi)-a_{h}(\chi, \psi), \quad \forall \chi, \psi \in V_{h} .
\end{aligned}
$$

In Section 6 we will give appropriate assumptions for $Q_{1}$ and $Q_{2}^{K}$ and the proof of Lemmas 5.1 and 5.2.

Lemma 5.1. There exists a constant $C=C(\sigma)$, such that

$$
\begin{array}{ll}
\left|\varepsilon_{h}(f, \chi)\right| \leq C \sum_{K} h_{K}\|f\|_{K}|\chi|_{1, K}, & \forall f \in L^{2}, \forall \chi \in V_{h}, \\
\left|\varepsilon_{h}(f, \chi)\right| \leq C \sum_{K} h_{K}^{2}|f|_{W^{1, p}(K)}|\chi|_{W^{1, q}(K)}, & \forall f \in W^{1, p}, \forall \chi \in V_{h},
\end{array}
$$

with $\int_{K} Q_{1} \chi=\int_{K} \chi$, for $\chi \in \mathbb{P}_{1}(K), K \in T_{h}$, and $\frac{1}{p}+\frac{1}{q}=1$ in (5.3).

Lemma 5.2. There exists a positive constant $C=C(\sigma, A)$ such that

$$
\begin{aligned}
& \left|\varepsilon_{a}(\psi, \chi)\right| \leq C \sum_{K} h_{K}|\psi|_{1, K}|\chi|_{1, K}, \quad \forall \chi, \psi \in V_{h} \\
& \left|\varepsilon_{a}\left(u_{h}, \chi\right)\right| \leq C h\left(\left|u_{h}-u\right|_{1, h}+h|u|_{2}\right)|\chi|_{1, h}+C h|u|_{2}|\chi-\psi|_{1, h},
\end{aligned}
$$

with $\chi \in V_{h}, \psi \in X_{h}^{0}$ and $A \in W^{2, \infty}$ in (5.5). 
Further, we have that the Poincaré-Friedrichs inequality holds in $V_{h}$, i.e.

$$
\|\chi\| \leq C\|\chi\|_{1, h}, \quad \forall \chi \in V_{h} .
$$

Remark 5.1. Clearly, (5.6) is satisfied when $V_{h}$ is $X_{h}^{0}$. In the case that $V_{h}$ is $S_{h}^{0}$, in view of [28, Prop. 4.13], we have that (5.6) also holds.

In view of Lemma 5.2 we can easily show that for $h$ sufficiently small, method (5.1) satisfies

$$
a_{h}(\chi, \chi) \geq \alpha_{1}|\chi|_{1, h}^{2}
$$

Then, due to (5.6), we have that the solution $u_{h}$ of (5.1) is uniquely defined. In the sequel we will show an optimal order $H^{1}$-norm error estimation.

Theorem 5.1. Let $u$ be the solution of (1.1) and $u_{h}$ the solution of (5.1). Then, there exists a constant $C$, independent of $h$, such that

$$
\left\|u-u_{h}\right\|_{1, h} \leq C h\|u\|_{2} .
$$

Proof. Due to the triangle inequality we have,

$$
\left\|u-u_{h}\right\|_{1, h} \leq\|u-\chi\|_{1, h}+\left\|u_{h}-\chi\right\|_{1, h}, \quad \forall \chi \in V_{h} .
$$

Thus, in view of the approximation properties (2.1) of $V_{h}$, it suffices to estimate the last part of (5.8). By the definition of $a(\cdot, \cdot)$ and $(1.2)$ we have,

$$
\left\|u_{h}-\chi\right\|_{1, h}^{2} \leq C\left|a\left(u_{h}-\chi, u_{h}-\chi\right)\right|, \quad \forall \chi \in V_{h} .
$$

Also, for every $\chi, \psi \in V_{h}$

$$
\left|a\left(u_{h}-\chi, \psi\right)\right|=\left|a\left(u_{h}-u, \psi\right)+a(u-\chi, \psi)\right| \leq\left|a\left(u_{h}-u, \psi\right)\right|+C\|u-\chi\|_{1, h}\|\psi\|_{1, h} .
$$

Therefore, choosing $\psi=u_{h}-\chi$ in the relation above and combining (5.9) we get

$$
\left\|u_{h}-\chi\right\|_{1, h}^{2} \leq C\left|a\left(u_{h}-u, u_{h}-\chi\right)\right|+C\|u-\chi\|_{1, h}\left\|u_{h}-\chi\right\|_{1, h}, \quad \forall \chi \in V_{h} .
$$

We can easily see that for every $\psi \in V_{h}$

$$
a\left(u-u_{h}, \psi\right)=a(u, \psi)-a_{h}\left(u_{h}, \psi\right)-\varepsilon_{a}\left(u_{h}, \psi\right)=\varepsilon_{h}(f, \psi)-\varepsilon_{a}\left(u_{h}, \psi\right) .
$$

Thus, choosing $\psi=u_{h}-\chi$ and combining Lemmas 5.1 and 5.2 and (5.10), for $h$ sufficiently small, we obtain

$$
\left\|u_{h}-\chi\right\|_{1, h} \leq C\|u-\chi\|_{1, h}+C h\|u\|_{2}, \quad \forall \chi \in V_{h} .
$$

In the sequel we will estimate the difference $u-u_{h}$ in the $L^{2}-$ norm.

Theorem 5.2. Let $u$ be the solution of (1.1) and $u_{h}$ the solution of (5.1). Assume that $f \in W^{1, p}, 1<p \leq 2$, $A \in W^{2, \infty}$ and

$$
\int_{K} \chi \mathrm{d} x=\int_{K} Q_{1} \chi \mathrm{d} x, \quad \forall K \in T_{h}, \forall \chi \in V_{h} .
$$

Then, there exists a constant $C$, independent of $h$, such that

$$
\left\|u-u_{h}\right\| \leq C h^{2}\left(\|u\|_{2}+\|f\|_{W^{1, p}}\right) .
$$


Proof. We consider the following auxiliary problem: Seek $\varphi \in H^{2}$ such that

$$
L \varphi=u-u_{h} \quad \text { in } \Omega \text { and } \varphi=0 \text { on } \partial \Omega .
$$

It is well known, $c f ., e . g .[17$, Chap. 4], that

$$
\|\varphi\|_{2} \leq C\left\|u-u_{h}\right\|
$$

Using (5.12) and Green's formula, we easily obtain

$$
\begin{aligned}
\left\|u-u_{h}\right\|^{2}=\left(u-u_{h}, L \varphi\right) & =a\left(u-u_{h}, \varphi\right)-\sum_{K}\left(A \nabla \varphi \cdot n, u-u_{h}\right)_{\partial K} \\
& =a\left(u-u_{h}, \varphi-\chi\right)+a\left(u-u_{h}, \chi\right)-\sum_{K}\left(A \nabla \varphi \cdot n, u-u_{h}\right)_{\partial K} \\
& =I+I I+I I I .
\end{aligned}
$$

The first term can obviously be estimated in the following way

$$
|I| \leq C\left\|u-u_{h}\right\|_{1, h}\|\varphi-\chi\|_{1, h}, \quad \forall \chi \in V_{h} .
$$

In the sequel we will estimate $I I$ and $I I I$. In view of $(5.11)$, we have

$$
I I=\varepsilon_{h}(f, \chi)-\varepsilon_{a}\left(u_{h}, \chi\right), \quad \forall \chi \in V_{h} .
$$

Thus, in view of Lemmas 5.1 and 5.2, we have

$$
\begin{aligned}
|I I| \leq & C h^{2} \sum_{K}|f|_{W^{1, p}(K)}|\chi|_{W^{1, q}(K)}+C h\left(\left|u_{h}-u\right|_{1, h}+h|u|_{2}\right)|\chi|_{1, h} \\
& +C h|u|_{2}|\chi-\psi|_{1, h}, \quad \forall \chi \in V_{h}, \forall \psi \in X_{h}^{0}
\end{aligned}
$$

with $\frac{1}{p}+\frac{1}{q}=1$. Next, let $I_{h}^{c}$ be the Lagrange interpolant $I_{h}^{c}: H^{2} \cap H_{0}^{1} \rightarrow X_{h}^{0}$. It is well known that

$$
\left\|I_{h}^{c} v-v\right\|_{1} \leq C h\|v\|_{2}, \quad \forall v \in H^{2} \cap H_{0}^{1} .
$$

Choosing now $\psi=I_{h}^{c} \varphi$ in (5.16) and using the estimation above we obtain

$$
\begin{aligned}
|I I| \leq & C h^{2} \sum_{K}|f|_{W^{1, p}(K)}|\chi|_{W^{1, q}(K)}+C h\left(\left|u_{h}-u\right|_{1, h}+h|u|_{2}\right)|\chi|_{1, h} \\
& +C h|u|_{2}\left(|\chi-\varphi|_{1, h}+h\|\varphi\|_{2}\right), \quad \forall \chi \in V_{h} .
\end{aligned}
$$

Let us now introduce the operator $\mathcal{M}_{\ell}^{\mu}$ as the orthogonal projection from $L^{2}(\ell)$ to $\mathbb{P}_{\mu}$, defined by

$$
\int_{\ell} w \mathcal{M}_{\ell}^{\mu} g \mathrm{~d} s=\int_{\ell} w g \mathrm{~d} s, \quad \forall w \in \mathbb{P}_{\mu}
$$

with $\ell$ a line segment of $\mathbb{R}^{2}$ and $\mathbb{P}_{\mu}$ the set of polynomials of two variables of degree at most $\mu \in \mathbb{N}$. Then according to [14, Lem. 3], for $K \in T_{h}$, we have

$$
\left|\int_{e} \varphi\left(g-\mathcal{M}_{e}^{0} g\right) \mathrm{d} s\right| \leq C h_{K}|\varphi|_{1, K}|g|_{1, K}, \quad \forall e \in E_{h}(K), \forall \varphi, g \in H^{1}(K) .
$$


Also, using the fact that $u, \varphi \in H^{2}$, and for any $e \in E_{h}$,

$$
\begin{gathered}
\int_{e}\left(\left.\chi\right|_{K_{1}}-\left.\chi\right|_{K_{2}}\right) \mathrm{d} s=0, \quad \forall \chi \in V_{h}, \text { if } e=K_{1} \cap K_{2}, K_{1}, K_{2} \in T_{h}, \\
\int_{e} \chi \mathrm{d} s=0, \quad \forall \chi \in V_{h}, \text { if } e \in E_{h} \backslash E_{h}^{\text {in }},
\end{gathered}
$$

we obtain, cf., e.g. [14],

$$
\sum_{K}\left(A \nabla \varphi \cdot n, u-u_{h}\right)_{\partial K}=\sum_{K} \sum_{e \in E_{h}(K)}\left(A \nabla \varphi \cdot n-\mathcal{M}_{e}^{0}((A \nabla \varphi) \cdot n), u-u_{h}\right)_{e} .
$$

Hence, in view of (5.19), we get

$$
|I I I|=\left|\sum_{K}\left(A \nabla \varphi \cdot n, u-u_{h}\right)_{\partial K}\right| \leq C h|\varphi|_{2}\left\|u-u_{h}\right\|_{1, h} .
$$

Next, let $I_{h}$ be the Lagrange interpolant $I_{h}: H^{2} \cap H_{0}^{1} \rightarrow V_{h}$. It is known that

$$
\begin{aligned}
\left\|I_{h} v-v\right\|_{1, h} & \leq C h\|v\|_{2}, \quad \forall v \in H^{2} \cap H_{0}^{1}, \\
\left\|I_{h} v\right\|_{W^{1, q}(K)} & \leq C\|v\|_{2, K}, \quad \forall v \in H^{2} \cap H_{0}^{1}, 2 \leq q<\infty, K \in T_{h},
\end{aligned}
$$

cf., e.g. [13, Th. 16.1]. Thus, choosing $\chi=I_{h} \varphi$ in (5.15) and (5.17) and combining (5.7), (5.13), (5.14), (5.20) and (5.21) we obtain the desired estimate.

\section{Auxiliary Results}

In this section we will give appropriate assumptions for operators $Q_{1}$ and $Q_{2}^{K}$ and prove Lemmas 5.1 and 5.2 of the previous section.

We consider a linear operator $Q_{1},\left.Q_{1}\right|_{K}: \mathbb{P}_{1}(K) \rightarrow L^{2}(K)$, with $K \in T_{h}$, satisfying $\int_{K} Q_{1} c=\int_{K} c$, for all constants c and $K \in T_{h}$ and also

$$
\left\|\chi-Q_{1} \chi\right\|_{L^{q}(K)} \leq h_{K}|\chi|_{W^{1, q}(K)}, \quad \forall \chi \in V_{h}, 1 \leq q<\infty .
$$

We can easily see that the operators $Q_{1}$ considered in (3.2), (3.6), (4.9) and (4.13), satisfy these assumptions. In addition, in view of Remarks 3.1 and 4.2 , these operators may satisfy:

$$
\int_{K} \chi \mathrm{d} x=\int_{K} Q_{1} \chi \mathrm{d} x, \quad \forall K \in T_{h}, \forall \chi \in V_{h}
$$

Also, we consider a linear operator $Q_{2}^{K},\left.Q_{2}^{K}\right|_{e}: \mathbb{P}_{1}(K) \rightarrow L^{2}(e)$, with $K \in T_{h}$ and $e$ a side of $K$, satisfying the following properties:

$$
\begin{aligned}
\left.Q_{2}^{K} c\right|_{e} & =c, & & \text { for all constants } c, e \text { a side of } K, \\
\int_{e} \chi \mathrm{d} s & =\int_{e} Q_{2}^{K} \chi \mathrm{d} s, & & \forall \chi \in \mathbb{P}_{1}(K), e \text { a side of } K, \\
\left\|Q_{2}^{K} \chi\right\|_{L^{\infty}(e)} & \leq\|\chi\|_{L^{\infty}(e)}, & & \forall \chi \in V_{h}+X_{h}^{0} ; \\
\left.Q_{2}^{K} \chi\right|_{e} & =\left.Q_{2}^{K_{2}} \chi\right|_{e}, & & \forall \chi \in V_{h}+X_{h}^{0}, e=K_{1} \cap K_{2}, K_{1}, K_{2} \in T_{h}, \\
\left.Q_{2}^{K} \chi\right|_{e} & =0, & & \forall \chi \in V_{h}+X_{h}^{0}, K \in T_{h}, e \in E_{h} \backslash E_{h}^{\text {in }} .
\end{aligned}
$$


We can easily see that the operators $Q_{2}^{K}$ defined in (4.11) and (4.15) and the restrictions of $\left.Q_{1}\right|_{e}$, on a side $e$, defined by (3.2) and (3.6), satisfy all the above assumptions. Next, we will show the following auxiliary lemma.

Lemma 6.1. Let $K$ be a triangle and e a side of $K$. Then for $\varphi \in H^{1}(K)$ there exists a constant $C$ independent of $K$ such that

$$
\left|\int_{e} \varphi\left(\chi-Q_{2}^{K} \chi\right) \mathrm{d} s\right| \leq C(\sigma) h_{K}|\varphi|_{1, K}|\chi|_{1, K}, \quad \forall \chi \in \mathbb{P}_{1}(K), \forall \varphi \in H^{1}(K)
$$

Proof. In view of (6.3) and (6.4), we have

$$
\int_{e} \varphi\left(\chi-Q_{2}^{K} \chi\right) \mathrm{d} s=\int_{e}\left(\varphi-c_{1}\right)\left(\chi-c_{2}-Q_{2}^{K}\left(\chi-c_{2}\right)\right) \mathrm{d} s, \forall \chi \in \mathbb{P}_{1}(K), \forall \varphi \in H^{1}(K),
$$

for all constants $c_{1}, c_{2} \in \mathbb{R}, K \in T_{h}$ and $e \in E_{h}(K)$. Using now in the relation above, (6.5) and a local inverse inequality, we get for all constants $c_{1}, c_{2} \in \mathbb{R}, \chi \in \mathbb{P}_{1}(K)$ and $\varphi \in H^{1}(K)$,

$$
\begin{aligned}
& \left|\int_{e} \varphi\left(\chi-Q_{2}^{K} \chi\right) \mathrm{d} s\right| \leq\left\|\varphi-c_{1}\right\|_{L^{2}(e)}\left\|\chi-c_{2}-Q_{2}^{K}\left(\chi-c_{2}\right)\right\|_{L^{2}(e)} \\
& \leq h_{e}^{1 / 2}\left\|\varphi-c_{1}\right\|_{L^{2}(e)}\left\|\chi-c_{2}-Q_{2}^{K}\left(\chi-c_{2}\right)\right\|_{L^{\infty}(e)} \\
& \leq C h_{e}^{1 / 2}\left\|\varphi-c_{1}\right\|_{L^{2}(e)}\left\|\chi-c_{2}\right\|_{L^{\infty}(e)} \leq C\left\|\varphi-c_{1}\right\|_{L^{2}(e)}\left\|\chi-c_{2}\right\|_{L^{2}(e)}
\end{aligned}
$$

with $h_{e}=|e|$. In view of the Bramble-Hilbert lemma and a standard homogeneity argument, we can easily show

$$
\inf _{c \in \mathbb{R}}\|\varphi-c\|_{L^{2}(e)} \leq C(\sigma) h_{e}^{1 / 2}|\varphi|_{1, K}, \quad \forall \varphi \in H^{1}(K)
$$

Finally, combining this with (6.8) we obtain the desired estimate.

In the sequel we will prove Lemmas 5.1 and 5.2.

Proof of Lem. 5.1. Due to (6.1), (5.2) follows easily. Let now $C_{K}(f)=\int_{B} f \omega_{K} \mathrm{~d} x$, where $B$ is a ball in $\mathbb{R}^{2}$, satisfying $B \subset \subset K$ and $\omega_{K}$ a cut-off function supported in $\bar{B}$, cf. [3, Def. 4.1.3]. Then, according to the Bramble-Hilbert lemma, in the form given in [3, Lem. 4.3.8], we obtain

$$
\left\|f-C_{K}(f)\right\|_{L^{p}(K)} \leq C(\sigma) h_{K}|f|_{W^{1, p}(K)}, \quad \forall f \in W^{1, p}(K), p \geq 1
$$

Since $C_{K}(f)$ is constant over $K$, due to $(6.2)$, we have

$$
\left(f, \chi-Q_{1} \chi\right)_{K}=\left(f-C_{K}(f), \chi-Q_{1} \chi\right)_{K}, \quad \forall \chi \in V_{h}
$$

Thus, due to this, (6.9) and (6.1), we get for every $\chi \in V_{h}$

$$
\left|\left(f, \chi-Q_{1} \chi\right)_{K}\right|=\left|\left(f-C_{K}(f), \chi-Q_{1} \chi\right)_{K}\right| \leq C h_{K}^{2}|f|_{W^{1, p}(K)}|\chi|_{W^{1, q}(K)},
$$

with $\frac{1}{p}+\frac{1}{q}=1$, which concludes the proof of (5.3).

Proof of Lem. 5.2. First we will show (5.4). In view of Green's formula, we have

$$
\varepsilon_{a}(\psi, \chi)=\sum_{K}\left(L \psi, \chi-Q_{1} \chi\right)_{K}+\sum_{K}\left(A \nabla \psi \cdot n, \chi-Q_{2}^{K} \chi\right)_{\partial K}=I+I I
$$


For the first term we have from (6.1),

$$
|I| \leq C \sum_{K}\|L \psi\|_{K}\left\|\chi-Q_{1} \chi\right\|_{K} \leq C \sum_{K} h_{K}|\psi|_{1, K}|\chi|_{1, K} .
$$

The bound for $I I$ follows at once from Lemma 6.1 since $|A \nabla \psi \cdot n|_{1, K} \leq C|\psi|_{1, K}$.

We now turn to (5.5). Let $\psi=u_{h}$ in (6.10) and $(\nabla A)_{K}$ be the average over $K$, then in view of $(6.2)$ we have for every $\chi \in V_{h}$

$$
\left.\left(L u_{h}, \chi-Q_{1} \chi\right)_{K}=\left(\left[\nabla A-(\nabla A)_{K}\right] \nabla u_{h}, \chi-Q_{1} \chi\right)\right)_{K} \leq C h_{K}^{2}\left|u_{h}\right|_{1, K}|\chi|_{1, K},
$$

from which we easily obtain the desired bound for $I$. Let now $\bar{A}_{e}=A\left(m_{e}\right)$ where $m_{e}$ is the midpoint of the edge $e$. We will show that for every $\chi \in V_{h}$ and $\psi \in X_{h}^{0}$

$$
\begin{aligned}
I I= & \sum_{K}\left(\left(A-\bar{A}_{e}\right) \nabla\left(u_{h}-u\right) \cdot n, \chi-Q_{2}^{K} \chi\right)_{\partial K} \\
& +\sum_{K}\left(\left(A-\bar{A}_{e}\right) \nabla u \cdot n, \chi-\psi-Q_{2}^{K}(\chi-\psi)\right)_{\partial K} .
\end{aligned}
$$

Provided that this holds, we may apply Lemma 6.1 and estimates

$$
\begin{aligned}
\left|\left(A-\bar{A}_{e}\right) \nabla\left(u_{h}-u\right)\right|_{1, K} & \leq C\left(\left|u_{h}-u\right|_{1, K}+h_{K}|u|_{2, K}\right), \\
\left|\left(A-\bar{A}_{e}\right) \nabla u\right|_{1, K} & \leq C\left(|u|_{1, K}+h_{K}|u|_{2, K}\right),
\end{aligned}
$$

to obtain

$$
\begin{aligned}
|I I| & \leq C \sum_{K} h_{K}\left(\left|u_{h}-u\right|_{1, K}+h_{K}|u|_{2}\right)|\chi|_{1, K}+\sum_{K} h_{K}\left(|u|_{1, K}+h_{K}|u|_{2}\right)|\chi-\psi|_{1, K} \\
& \leq C h\left(\left|u_{h}-u\right|_{1, h}+h|u|_{2}\right)|\chi|_{1, h}+C h|u|_{2}|\chi-\psi|_{1, h}, \quad \forall \chi \in V_{h}, \psi \in X_{h}^{0},
\end{aligned}
$$

which gives the desired estimate for $I I$. Therefore it remains to prove (6.11). For this we will show, for every $\psi \in X_{h}^{0}$

$$
\sum_{K}\left(A \nabla u \cdot n, \psi-Q_{2}^{K} \psi\right)_{\partial K}=\sum_{K}\left(\bar{A}_{e} \nabla u \cdot n, \psi-Q_{2}^{K} \psi\right)_{\partial K}=0
$$

In the first sum we have by Green's formula for every $\psi \in X_{h}^{0}$

$$
\sum_{K}(A \nabla u \cdot n, \psi)_{\partial K}=\sum_{K}(A \nabla u, \nabla \psi)_{K}-(L u, \psi)_{K}=(A \nabla u, \nabla \psi)-(L u, \psi)=0 .
$$

In addition, $\sum_{K}\left(A \nabla u \cdot n, Q_{2}^{K} \psi\right)_{\partial K}=0$ because $Q_{2}^{K} \psi$ is piecewise constant on each interior edge $e$ and $A \nabla u \cdot n$ is continuous across $e$ (in the trace sense), and $Q_{2}^{K} \psi=0$ on $\partial \Omega$. Since the first sum in (6.12) vanishes for each smooth $A$, and is continuous in $A$ on $L^{1}(\cup \partial K)$ the second sum is the limit of sums with a smooth $A$ and therefore also vanishes. Finally, since $\bar{A}_{e} \nabla u_{h} \cdot n$ is constant on each $e$, in view of (6.4) we have

$$
\sum_{K}\left(\bar{A}_{e} \nabla u_{h} \cdot n, \chi-Q_{2}^{K} \chi\right)_{\partial K}=0, \quad \forall \chi \in V_{h} .
$$


Remark 6.1. It is known, cf., e.g. [17, Chap. 5.2], that if $f \in L^{p}, 1<p<2$, the solution $u$ of (1.1) is an element of $W^{2, p}$ and by imbedding, $u \in H^{s}$, where $H^{s}$ is the fractional order Sobolev space, with $s=3-2 / p$, cf. [1, Chap. VII, Th. 7.58]. For (1.5) to make sense, we need $s>3 / 2$ and thus $p>4 / 3$. Therefore, one can prove analogous results to Theorems 5.1 and 5.2 , that is,

$$
\left\|u-u_{h}\right\|_{1, h} \leq C h^{2-2 / p}\|u\|_{W^{2, p}}
$$

and, if in addition $f \in W^{1, p}$, since then $f \in L^{2}$, we have

$$
\left\|u-u_{h}\right\| \leq C h^{2}\left(\|u\|_{2}+\|f\|_{W^{1, p}}\right) .
$$

To obtain these estimations we need to modify Lemma 6.1 and prove that

$$
\left|\left(\varphi, \chi-Q_{2}^{K} \chi\right)_{e}\right| \leq C h_{K}|\varphi|_{W^{1, p}(K)}|\chi|_{W^{1, q}(K)}, \forall \varphi \in W^{1, p}(K), \forall \chi \in \mathbb{P}_{1}(K)
$$

where $\frac{1}{p}+\frac{1}{q}=1$. Also, we need the local inverse inequality

$$
|\psi|_{W^{1, q}(K)} \leq C h_{K}^{1-2 / p}|\psi|_{1, K}, \quad \forall \psi \in V_{h}
$$

Further, using the interpolation theory of Sobolev spaces, cf., e.g. [3, Chap. 12], we have

$$
\inf _{\chi \in V_{h}}\|u-\chi\|_{1, h} \leq C h^{s-1}\|u\|_{H^{s}}, \quad 1<s<2 .
$$

Following the proof of Theorems 5.1 and 5.2 and combining (6.15)-(6.17), we can derive estimations (6.13) and (6.14).

Acknowledgements. The author would like to thank Professor Charalambos Makridakis for suggesting the problem and Professors Georgios Akrivis, Charalambos Makridakis and Vidar Thomée for contributing several valuable remarks. Also the author would like to thank Professor Michel Crouzeix for his suggestions and in particular for suggesting to use Lemma 6.1 and the necessary properties for the operator $Q_{2}^{K}$.

\section{REFERENCES}

[1] R.A. Adams, Sobolev Spaces. Academic Press, New York (1975).

[2] R.E. Bank and D.J. Rose, Some error estimates for the box method. SIAM J. Numer. Anal. 24 (1987) 777-787.

[3] S.C. Brenner and L.R. Scott, The Mathematical Theory of Finite Element Methods. Springer-Verlag, New York (1994).

[4] B. Brighi, M. Chipot and E. Gut, Finite differences on triangular grids. Numer. Methods Partial Differential Equations 14 (1998) 567-579.

[5] Z. Cai, On the finite volume element method. Numer. Math. 58 (1991) 713-735.

[6] S. Champier, T. Gallouët and R. Herbin, Convergence of an upstream finite volume scheme for a nonlinear hyperbolic equation on a triangular mesh. Numer. Math. 66 (1993) 139-157.

[7] P. Chatzipantelidis, A finite volume method based on the Crouzeix-Raviart element for elliptic PDE's in two dimensions. Numer. Math. 82 (1999) 409-432.

[8] P. Chatzipantelidis, R.D. Lazarov and V. Thomée, Error estimates for the finite volume element method for parabolic pde's in convex polygonal domains. In preparation.

[9] P. Chatzipantelidis and R.D. Lazarov, The finite volume element method in nonconvex polygonal domains. To appear in Proceedings of the Third International Symposium on Finite Volumes for Complex Applications, Hermes Science Publications, Paris (2002).

[10] P. Chatzipantelidis, Ch. Makridakis and M. Plexousakis, A-posteriori error estimates of a finite volume scheme for the Stokes equations. In preparation.

[11] S.H. Chou, Analysis and convergence of a covolume method for the generalized Stokes problem. Math. Comp. 66 (1997) $85-104$. 
[12] S.H. Chou and Q. Li, Error estimates in $L^{2}, H^{1}$ and $L^{\infty}$ in covolume methods for elliptic and parabolic problems: a unified approach. Math. Comp. 69 (2000) 103-120.

[13] P.G. Ciarlet, Basic Error Estimates for Elliptic Problems. Handbook of Numerical Analysis, Vol. II, North-Holland, Amsterdam (1991) 17-351.

[14] M. Crouzeix and P.-A. Raviart, Conforming and nonconforming finite element methods for solving the stationary Stokes equation I. RAIRO Anal. Numér. 7 (1973) 33-76.

[15] R.E. Ewing, R.D. Lazarov and Y. Lin, Finite Volume Element Approximations of Nonlocal Reactive Flows in Porous Media. Numer. Methods Partial Differential Equations 16 (2000) 285-311.

[16] R. Eymard, T. Gallouët and R. Herbin, Finite Volume Methods. Handbook of Numerical Analysis, Vol. VII, North-Holland, Amsterdam (2000).

[17] P. Grisvard, Elliptic Problems in Nonsmooth Domains. Pitman, Massachusetts (1985).

[18] W. Hackbusch, On first and second order box schemes. Comput. 41 (1989) 277-296.

[19] H. Jianguo and X. Shitong, On the finite volume element method for general self-adjoint elliptic problems. SIAM J. Numer. Anal. 35 (1998) 1762-1774.

[20] S. Kang and D.Y. Kwak, Error estimate in $L^{2}$ of a covolume method for the generalized Stokes Problem. Proceedings of the eight KAIST Math Workshop on Finite Element Method, KAIST (1997) 121-139.

[21] G. Kossioris, Ch. Makridakis and P.E. Souganidis, Finite volume schemes for Hamilton-Jacobi equations. Numer. Math. 83 (1999) 427-442.

[22] F. Liebau, The finite volume element method with quadratic basis functions. Comput. 57 (1996) 281-299.

[23] I.D. Mishev, Finite volume element methods for non-definite problems. Numer. Math. 83 (1999) 161-175.

[24] K.W. Morton, Numerical Solution of Convection-Diffusion Problems. Chapman \& Hall, London (1996).

[25] M. Plexousakis and G.E. Zouraris, High-order locally conservative finite volume-type approximations of one dimensional elliptic problems. Technical Report, TRITA-NA-0138, NADA, Royal Institute of Technology, Sweden.

[26] H.-G. Roos, M. Stynes and L. Tobiska, Numerical Methods for Singularly Perturbed Differential Equations. Springer-Verlag, Berlin (1996).

[27] T. Schmidt, Box schemes on quadrilateral meshes. Comput. 51 (1994) 271-292.

[28] R. Temam, Navier-Stokes Equations. North-Holland, Amsterdam (1979).

[29] A. Weiser and M.F. Wheeler, On convergence of Block-Centered finite differences for elliptic problems. SIAM J. Num. Anal. 25 (1988) 351-375.

To access this journal online: www.edpsciences.org 